\title{
Solving a Class of Self-adjoint Differential Equations of the Fourth Order and its Algorithms in MATLAB
}

\author{
Mehdi Delkhosh \\ Islamic Azad University, Bardaskan Branch, Department of Mathematics, Bardaskan, Iran \\ *Corresponding Author: mehdidelkhosh@yahoo.com
}

Copyright (C) 2013 Horizon Research Publishing All rights reserved.

\begin{abstract}
In many of applied sciences, various Self-adjoint differential equations are generated, where, methods for their solution is very complex. Usually, numerical methods used to solve them. Leighton et al were investigated oscillation properties of solutions of self-adjoint differential equations of the fourth order, with specific conditions. In this paper, we use a new method for the solving a class of Self-adjoint differential equations of the fourth order. We use a variable change in the equation, and then obtain an analytical solution for the equation with a specific condition. Because in this method, an analytical solution is obtained, therefore, it is not necessary to use numerical methods to solve the problem.
\end{abstract}

Keywords Self-adjoint Differential Equation, Ordinary Differential Equation, Linear Differential Equation, Wronskian

\section{Introduction}

In many of applied sciences, often appear equations of type Self-adjoint equations, where methods their solution are very complex. Usually, numerical methods used to solve them, which are contained several errors $[7,9,11,13$, 14, and 16].

There are several methods for solving equations, there one of which can be seen in the literature $[4,5,6,12,15,20$, $22]$, which they used change variables and complex methods.

In paper [19] W. Leighton and Z. Nehari, investigated oscillation properties of solutions of self-adjoint differential equations of the fourth order

$$
\left(r(x) y^{\prime \prime}\right)^{\prime \prime}+\left(q(x) y^{\prime}\right)^{\prime}+p(x) y=0
$$

with particular attention to the cases where the middle term is missing, $r(x)>0$ and $p(x)$ does not change sign.

In paper [8] J.H. Barrett, one of these particular cases $\left(r(x) y^{\prime \prime}\right)^{\prime \prime}+p(x) y=0 \quad(r(x)$ and $p(x)$ positive and continuous on $\lceil 0, \infty\rceil)$ be pursued further with the object of paralleling the known theory of second order equation $\left(r(x) y^{\prime}\right)^{\prime}+p(x) y=0$ with positive and continuous coefficients (e.g., see $[10,18,20,21])$.

In this paper, for solving analytical some Self-adjoint Differential Equations of the Fourth Order, we get a method which with variable change in problem, and then we obtain an analytical solutions.

Before going to the main point, we start to introduce three following equations:

\subsection{Self-adjoint Differential Equations of the Fourth Order}

A four order linear homogeneous differential equation is called Self-adjoint if And only if it has the following form $[2,3,8,12,15,17,19$, and 22]:

$$
\left(r(x) y^{\prime \prime}\right)^{\prime \prime}+\left(q(x) y^{\prime}\right)^{\prime}+p(x) y=0
$$

where $r(x)>0$ on $(a, b)$ and $r(x), q(x)$ are continuous functions and differentiable on $[a, b]$.

\subsection{Wronskian}

The Wronskian of two functions $\mathrm{f}$ and $\mathrm{g}$ is [1, 2, and 3]

$$
W(x)=W(f, g)=f^{\prime} g-f g^{\prime} .
$$

More generally, for $\mathrm{n}$ real- or complex- valued functions $f_{1}, f_{2}, \ldots, f_{n}$, which are $\mathrm{n}-1$ times differentiable on an interval $\mathrm{I}$, the Wronskian $W(x)=W\left(f_{1}, \ldots, f_{n}\right)$ as a function on $\mathrm{I}$ is defined by

$$
W(x)=\left|\begin{array}{ccc}
f_{1} & \cdots & f_{n} \\
\vdots & \ddots & \vdots \\
f_{1}^{(n-1)} & \cdots & f_{n}^{(n-1)}
\end{array}\right|
$$

That is, it is the determinant of the matrix constructed by placing the functions in the first row, the first derivative of each function in the second row, and so on through the (n-1)st derivative, thus forming a square matrix sometimes called a fundamental matrix.

When the functions $f_{i}$ are solutions of a linear 
differential equation, the Wronskian can be found explicitly using Abel's identity, even if the functions $f_{i}$ are not known explicitly.

Theorem 1: if $P(x) y^{\prime \prime}+Q(x) y^{\prime}+R(x) y=0$ then

$$
W(x)=e^{-\int \frac{Q}{P} d x}
$$

Proof: let two solution of equation by $y_{1}$ and $y_{2}$, then, since these solutions satisfy the equation, we have

$$
\begin{aligned}
& P y_{1}^{\prime \prime}+Q y_{1}^{\prime}+R y_{1}=0 \\
& P y_{2}^{\prime \prime}+Q y_{2}^{\prime}+R y_{2}=0
\end{aligned}
$$

Multiplying the first equation by $y_{2}$, the second by $y_{1}$, and subtracting, we find

$$
P .\left(y_{1} y_{2}^{\prime \prime}-y_{2} y_{1}^{\prime \prime}\right)+Q \cdot\left(y_{1} y_{2}^{\prime}-y_{2} y_{1}^{\prime}\right)=0
$$

Since Wronskian is given by $W=y_{1} y_{2}^{\prime}-y_{2} y^{\prime}$ thus

$$
P . \frac{d W}{d x}+Q . W=0
$$

Solving, we obtain an important relation known as Abel's identity, given by

$$
W(x)=e^{-\int \frac{Q}{P} d x}
$$

\section{Solving a Class of Self-adjoint Differential Equations of the Fourth Order}

Theorem 2 (Main Theorem): If, a Self-adjoint Differential Equation of the Fourth Order:

$$
\left(r(x) y^{\prime \prime}\right)^{\prime \prime}+\left(q(x) y^{\prime}\right)^{\prime}+p(x) y=0
$$

verify in the conditions:

$$
\begin{gathered}
q(x)=2 r^{\prime \prime}-\frac{3}{2} \frac{r^{\prime 2}}{r} \\
p(x)=\frac{1}{8}\left(2 q^{\prime \prime}-\frac{r^{\prime}}{r} q^{\prime}\right)
\end{gathered}
$$

Then, the answer to equation will be

$$
y(x)=\frac{1}{\sqrt{r(x)}}\left(C_{3} x^{3}+C_{2} x^{2}+C_{1} x+C_{0}\right)
$$

where $C_{3}, C_{2}, C_{1}, C_{0}$ are arbitrary real numbers.

Proof: we show that Self-adjoint differential equation of the fourth order (1) is changeable to two linear differential equations.

$$
\begin{aligned}
& \left(r y^{\prime \prime}\right)^{\prime \prime}+\left(q y^{\prime}\right)^{\prime}+p y=0 \\
& \Rightarrow r y^{(4)}+2 r^{\prime} y^{\prime \prime \prime}+\left(r^{\prime \prime}+q\right) y^{\prime \prime}+q^{\prime} y^{\prime}+p y=0
\end{aligned}
$$

then

$$
y^{(4)}+\frac{2 r^{\prime}}{r} y^{\prime \prime \prime}+\frac{r^{\prime \prime}+q}{r} y^{\prime \prime}+\frac{q^{\prime}}{r} y^{\prime}+\frac{p}{r} y=0
$$

By replacing of change variable $y=u(x) \cdot v(x)$, where $u(x)$ and $v(x)$ are continuous and differentiable functions, we obtain

$$
\begin{aligned}
& v \cdot u^{(4)}+\left[4 v^{\prime}+\frac{2 r^{\prime}}{r} v\right] u^{\prime \prime \prime} \\
& +\left[6 v^{\prime \prime}+\frac{6 r^{\prime}}{r} v^{\prime}+\frac{r^{\prime \prime}+q}{r} v\right] u^{\prime \prime} \\
& +\left[4 v^{\prime \prime \prime}+\frac{6 r^{\prime}}{r} v^{\prime \prime}+2 \frac{r^{\prime \prime}+q}{r} v^{\prime}+\frac{q^{\prime}}{r} v\right] u^{\prime} \\
& +\left[v^{(4)}+\frac{2 r^{\prime}}{r} v^{\prime \prime \prime}+\frac{r^{\prime \prime}+q}{r} v^{\prime \prime}+\frac{q^{\prime}}{r} v^{\prime}+\frac{p}{r} v\right] u=0
\end{aligned}
$$

Now, we assume that the coefficient $u^{\prime \prime \prime}$ is zero, and we can calculate the value $v(x)$, i.e.

$$
4 v^{\prime}+\frac{2 r^{\prime}}{r} v=0
$$

Now, corresponding to equations (7) and (4), we have

$$
v(x)=e^{-\frac{1}{2} \int \frac{r^{\prime}}{r} d x}=(r(x))^{-\frac{1}{2}}=\frac{1}{\sqrt{r(x)}}=\sqrt{W(x)}
$$

where $W(x)$ is Wronskian.

Now, by using Equations (6), (8) and assuming that the coefficients $u^{\prime \prime}, u^{\prime}, u$ are zero, we get the condition that if an equation is also conditions; can be solved with this method:

$$
\begin{gathered}
6 v^{\prime \prime}+\frac{6 r^{\prime}}{r} v^{\prime}+\frac{r^{\prime \prime}+q}{r} v=0 \\
4 v^{\prime \prime \prime}+\frac{6 r^{\prime}}{r} v^{\prime \prime}+2 \frac{r^{\prime \prime}+q}{r} v^{\prime}+\frac{q^{\prime}}{r} v=0 \\
v^{(4)}+\frac{2 r^{\prime}}{r} v^{\prime \prime \prime}+\frac{r^{\prime \prime}+q}{r} v^{\prime \prime}+\frac{q^{\prime}}{r} v^{\prime}+\frac{p}{r} v=0
\end{gathered}
$$

By replacing Equation (8) in equations (9), (11), we obtain

$$
-2 r^{\prime \prime} r^{-\frac{1}{2}}+\frac{3}{2} r^{\prime 2} r^{-\frac{3}{2}}+q r^{-\frac{1}{2}}=0
$$

and

$$
\begin{aligned}
-\frac{1}{2} r^{(4)} r^{-\frac{1}{2}}+r^{\prime} r^{\prime \prime \prime} r^{-\frac{3}{2}}+\frac{3}{4} r^{\prime \prime 2} r^{-\frac{3}{2}} & \\
- & \frac{9}{4} r^{\prime 2} r^{\prime \prime} r^{-\frac{5}{2}}+\frac{15}{16} r^{\prime 4} r^{-\frac{7}{2}}+p r^{-\frac{1}{2}}=0
\end{aligned}
$$

By replacing Equation (8) in equations (10), both sides of the equation are zero.

Now, corresponding to equations (12) and (13), we have 


$$
\begin{gathered}
q(x)=2 r^{\prime \prime}-\frac{3}{2} \frac{r^{\prime 2}}{r} \\
p(x)=\frac{1}{16}\left(8 r^{(4)}-16 \frac{r^{\prime} r^{\prime \prime \prime}}{r}-12 \frac{r^{\prime 2}}{r}+36 \frac{r^{\prime 2} r^{\prime \prime}}{r^{2}}-15 \frac{r^{\prime 4}}{r^{3}}\right)
\end{gathered}
$$

Or

$$
\begin{gathered}
q(x)=2 r^{\prime \prime}-\frac{3}{2} \frac{r^{\prime 2}}{r} \\
p(x)=\frac{1}{8}\left(2 q^{\prime \prime}-\frac{r^{\prime}}{r} q^{\prime}\right)
\end{gathered}
$$

Thus, if in Self-adjoint differential equation of the fourth order (1), Equations (14) and (15) are established, we have:

$$
v \cdot u^{(4)}=0 \quad \Rightarrow u^{(4)}=0
$$

Or

$$
u(x)=C_{3} x^{3}+C_{2} x^{2}+C_{1} x^{1}+C_{0}
$$

where $C_{3}, C_{7}, C_{1}, C_{0}$ are arbitrary real numbers.

So, we have from Equations (8) and (16):

$$
\begin{aligned}
& v(x)=e^{-\frac{1}{2} \int \frac{r^{\prime}}{r} d x}=(r(x))^{-\frac{1}{2}}=\frac{1}{\sqrt{r(x)}}=\sqrt{W(x)} \\
& u(x)=C_{3} x^{3}+C_{2} x^{2}+C_{1} x^{1}+C_{0}
\end{aligned}
$$

And, the answer to Self-adjoint differential equation of the fourth order (1), will be

$$
y(x)=v(x) \cdot u(x)=\frac{1}{\sqrt{r(x)}}\left(C_{3} x^{3}+C_{2} x^{2}+C_{1} x+C_{0}\right)
$$

\section{Examples and Applications}

EX.1) Solve the equation

$$
\left(x^{2} y^{\prime \prime}\right)^{\prime \prime}+\left(-2 y^{\prime}\right)^{\prime}+0 \cdot y=0
$$

where $x \neq 0$.

Solution: By virtue of equations (1), we have

$$
r(x)=x^{2} \quad, \quad q(x)=-2, \quad p(x)=0
$$

Obviously, that equations (14) and (15) are established, i.e.

$$
\begin{gathered}
q(x)=2(2)-\frac{3}{2} \frac{(2 x)^{2}}{x^{2}}=4-6=-2 \\
p(x)=\frac{1}{8}\left(2.0-\frac{2 x}{x^{2}} .0\right)=0
\end{gathered}
$$

So, we have:

$$
\begin{aligned}
y(x) & =\frac{1}{\sqrt{x^{2}}}\left(C_{3} x^{3}+C_{2} x^{2}+C_{1} x+C_{0}\right) \\
& =C_{3} x^{2}+C_{2} x^{1}+C_{1}+C_{0} x^{-1}
\end{aligned}
$$

EX.2) Solve the equation

$$
\left((1+x)^{8} y^{\prime \prime}\right)^{\prime \prime}+\left(16(1+x)^{6} y^{\prime}\right)^{\prime}+24(1+x)^{4} y=0
$$

where $x \neq-1$.

Solution: By virtue of equations (1), we have

$r(x)=(1+x)^{8}, q(x)=16(1+x)^{6}, p(x)=24(1+x)^{4}$

Obviously, that equations (14) and (15) are established, i.e.

$$
\begin{aligned}
q(x) & =2.56(1+x)^{6}-\frac{3}{2} \frac{64(1+x)^{14}}{(1+x)^{8}} \\
& =112(1+x)^{6}-96(1+x)^{6}=16(1+x)^{6}
\end{aligned}
$$

$$
\begin{aligned}
p(x) & =\frac{1}{8}\left(2.480(1+x)^{4}-\frac{8(1+x)^{7}}{(1+x)^{8}} \cdot 96(1+x)^{5}\right) \\
& =\frac{1}{8}(960-768)(1+x)^{4}=24(1+x)^{4}
\end{aligned}
$$

So, we have:

$$
\begin{aligned}
y(x) & =\frac{1}{\sqrt{(1+x)^{8}}}\left(C_{3} x^{3}+C_{2} x^{2}+C_{1} x+C_{0}\right) \\
& =\frac{1}{(1+x)^{4}}\left(C_{3} x^{3}+C_{2} x^{2}+C_{1} x+C_{0}\right)
\end{aligned} .
$$

EX.3) Solve the equation

$$
\left(\sqrt{x} y^{\prime \prime}\right)^{\prime \prime}+\left(\frac{-7}{8 \sqrt{x^{3}}} y^{\prime}\right)^{\prime}+\frac{-231}{256 \sqrt{x^{7}}} y=0
$$

where $x \neq 0$.

Solution: By virtue of equations (1), we have

$$
r(x)=\sqrt{x} \quad, \quad q(x)=\frac{-7}{8 \sqrt{x^{3}}} \quad, \quad p(x)=\frac{-231}{256 \sqrt{x^{7}}}
$$

Obviously, those equations (14) and (15) are established, So, we have:

$$
\begin{aligned}
y(x) & =\frac{1}{\sqrt{\sqrt{x}}}\left(C_{3} x^{3}+C_{2} x^{2}+C_{1} x+C_{0}\right) \\
& =\frac{1}{\sqrt[4]{x}}\left(C_{3} x^{3}+C_{2} x^{2}+C_{1} x+C_{0}\right)
\end{aligned}
$$

\section{Algorithms in MATLAB}

\subsection{Algorithm for Finding the Functions}

Algorithm for finding the functions in equations (14) 
and (15) are true, is as follows:

First, in MATLAB $\Rightarrow$ Menu "File" $\Rightarrow$ Select "NEW" $\Rightarrow$ Select "M-File" $\Rightarrow$ The following function to copy in file, and then save the file to "adjoint4_find.m" name:

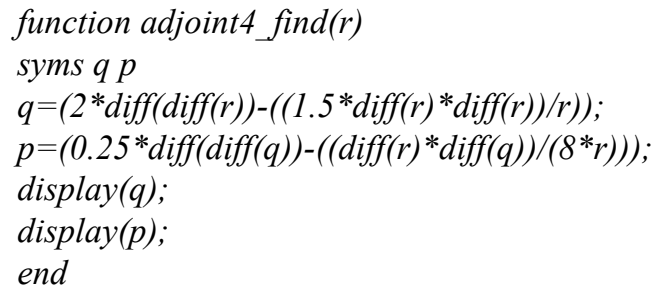

Thus, functions that are true in the equations (14) and (15) are calculated, the desired equation is as follows:

$$
\left((1+x)^{3} y^{\prime \prime}\right)^{\prime \prime}+\left(-\frac{3}{2}(1+x) y^{\prime}\right)^{\prime}+\frac{9}{16}(1+x) y=0
$$
i.e.

$$
y(x)=\frac{1}{\sqrt{(1+x)^{3}}}\left(C_{3} x^{3}+C_{2} x^{2}+C_{1} x+C_{0}\right)
$$

\subsection{Algorithm to Check the True Functions}

Algorithm to check the true functions in equations (14) and (15), is as follows:

First, in MATLAB $\Rightarrow$ Menu "File" $\Rightarrow$ Select "NEW" $\Rightarrow$ Select "M-File" $\Rightarrow$ The following function to copy in file, and then save the file to "adjoint4_test.m" name:

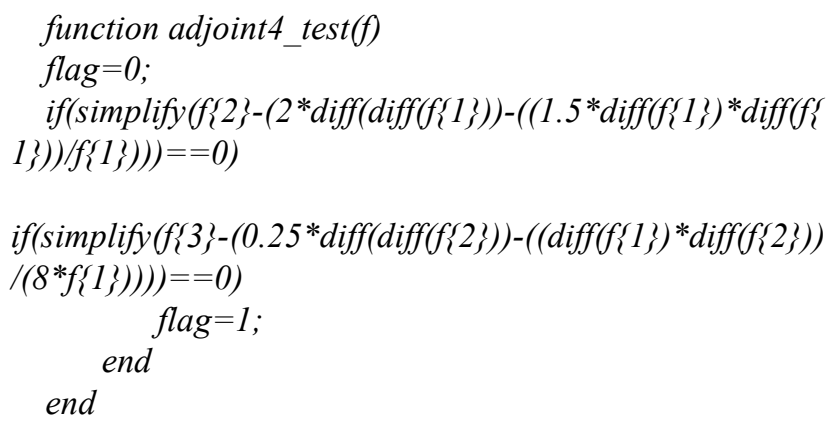

$$
\begin{aligned}
& \text { if }(\text { flag }==1) \\
& \quad \operatorname{display}\left({ }^{\prime} \mathrm{Yes}^{\prime}\right) \text {; } \\
& \text { else } \\
& \text { display('No'); } \\
& \text { end } \\
& \text { end }
\end{aligned}
$$

Secondly, in the window "Command Windows" of MATLAB, type the following commands:

$$
>>\text { syms } x
$$

$>>f\{1\}=$ "the intended function $r(x)$ ";

$>>f 2\}=$ "the intended function $q(x) "$;

$>>\{3\}=$ "the intended function $p(x)$ ";

$>>$ adjoint4_test $(f)$

EX.5) in the window "Command Windows" of MATLAB, type the following commands:

$$
\begin{aligned}
& >>\operatorname{syms} x \\
& >>f\{1\}=1 /(1+x) ; \\
& >>f\{2\}=5 /\left(2 *(1+x)^{\wedge} 3\right) ; \\
& >>f\{3\}=105 /\left(16^{*}(1+x)^{\wedge} 5\right) ; \\
& >>\text { adjoint4_test }(f)
\end{aligned}
$$

Then, key "ENTER"

$$
\begin{aligned}
& >>\text { yes } \\
& \quad \begin{array}{l}
\text { Thus, if } \\
r(x)=1 /(1+x), q(x)=5 / 2(1+x)^{\circ}, p(x)=105 / 16(1+x)^{\circ}
\end{array}
\end{aligned}
$$

, equations (14) and (15) are established, i.e. equation

$$
\left(1 /(1+x) y^{\prime \prime}\right)^{\prime \prime}+\left(5 / 2(1+x)^{3} y^{\prime}\right)^{\prime}+105 / 16(1+x)^{5} y=0
$$
is the answer to:

$$
y(x)=\sqrt{(1+x)}\left(C_{3} x^{3}+C_{2} x^{2}+C_{1} x+C_{0}\right) .
$$

\section{Conclusions}

The governing equation for stability analysis of a variable cross-section bar subject to variably distributed axial loads, dynamic analysis of multi-storey building, tall building and other systems are written in the form of a unified Self-adjoint equation. The key step in transforming the unified equation to Self-adjoint equation is the selection of $q(x)$ and $p(x)$ in equation (1).

Many difficult problems in the field of static and dynamic mechanics are solved by the unified equation proposed in this paper.

\section{REFERENCES}

[1] F.B. Hilderbrand, Advanced Calculus for Applications (2-ed), New Jersey, (1976).

[2] S.H. Javadpour, an Introduction to Ordinary and Partial Differential Equations, Iran, Alavi, (1993). 
[3] P.V. O’Neil, Advanced Engineering Mathematics (2-ed), California, Wadsworth, (1987).

[4] L. Qiusheng, C. Hong, L. Guiqing, Static and Dynamic analysis of straight bars with variable cross-section, Computer \& Structures Vol. 59, No. 6, (1996), p.1185-1191.

[5] L. Qiusheng, C. Hong, L. Guiqing, Analysis of Free Vibrations of Tall Buildings, Journal of Engineering Mechanics, Vol. 120, No. 9, (1994), p. 1861-1876.

[6] D. Demir, N. Bildik, A. Konuralp, A. Demir, The Numerical Solutions for the Damped Generalized Regularized Long-wave Equation by Variational Method, World Applied Sciences Journal (Special Issue of Applied Math): No. 13, (2011), p. 08-17, ISSN 1818-4952

[7] S.T. Mohyud-Din, Solutions of Nonlinear Differential Equations by Exp-function Method, World Applied Sciences Journal (Special Issue for Applied Math), No.7, (2009), p. 116-147, ISSN 1818-4952

[8] J.H. Barrett, Disconjugacy of a self-adjoint differential equation of the fourth order, the Office of Ordnance Research, U. S. Army, Contract DA-04-495-ORD-1088 with the University of Utah. Presented to the Amer. Math. Soc, January, (1960).

[9] M. Allame, N. Azad, Solution of Third Order Nonlinear Equation by Taylor Series Expansion, World Applied Sciences Journal, No 14, (2011), p. 59-62.

[10] Z. Nehari, Oscillation criteria for second-order linear differential equations, Trans. AMS, No. 85, (1958), p. 428-445.

[11] A. Borhanifar, M.M. Kabir, A. HosseinPour, A Numerical Method for Solution of the Heat Equation with Nonlocal Nonlinear Condition, World Applied Sciences Journal, No. 13, (2011), p. 2405-2409.

[12] Arfken, G. "Self-Adjoint Differential Equations." §9.1 in Mathematical Methods for Physicists, 3rd ed. Orlando, FL:
Academic Press, (1985), p. 497-509.

[13] N.H. Sweilam, A.M. Nagy, Numerical Solution of Fractional Wave Equation using Crank-Nicholson Method, World Applied Sciences Journal (Special Iss. of App. Math), No. 13, (2011), p. 71-75.

[14] M. Gülsu, Y. Öztürk, M. Sezer, Numerical Solution of Singular Integra-differential Equations with Cauchy Kernel, World Applied Sciences Journal , No. 13, (2011), p. 2420-2427, ISSN 1818-4952

[15] M. Gandarias, Weak self-adjoint differential equations, J. Phys. A: Math. Theor., No 44,(2011), 262001 (6pp)

[16] T. Mohyud-Din, A. Yildirim, M. Berberler, and M. Hosseini, Numerical Solution of Modified Equal Width Wave Equation, World Applied Sciences Journal, No. 8, (2010), p. 792-798, ISSN 1818-4952

[17] M. Delkhosh, The Conversion a Bessel's Equation to a Self-Adjoint Equation and Applications, World Applied Sciences Journal, No. 15, (2011), p. 1687-1691,

[18] J.H. Barrett, Disconjugacy of second-order linear differential equations with non-negative coefficients, Proc. AMS, No. 10, (1959), p. 552-561.

[19] W. Leighton, Z. Nehari, on the oscillation of solutions of self-ad joint linear differential equations of the fourth order Trans. AMS., No. 89, (1958), p. 325-377.

[20] M. Delkhosh, Analytic Solutions of Some Self-adjoint Equations by Using Variable Change Method And it's Applications, Journal of Applied Mathematics, Volume 2012, Article ID 180806, doi:10.1155/2012/180806

[21] M. Marletta, The growth order of a solution of a singular non-self-adjoint differential equation as a function of the spectral parameter, Integr. Equ. Oper. Theory, No. 72 (2012), p. 5-6.

[22] C. Byrne, Notes on Self-adjoint Differential Equations, http://faculty.uml.edu/ cbyrne/Sturm.pdf, (2009). 